\title{
Essais
}

Revue interdisciplinaire d'Humanités

Hors-série 3 | 2016

Usages critiques de Montaigne

\section{Ce que les Essais nous apprennent sur les impensés de la philosophie}

\section{Thomas Mollier}

\section{(2) OpenEdition}

1 Journals

Édition électronique

URL : http://journals.openedition.org/essais/6819

DOI : 10.4000/essais. 6819

ISSN : 2276-0970

Éditeur

École doctorale Montaigne Humanités

\section{Édition imprimée}

Date de publication : 1 novembre 2016

Pagination : 68-83

ISBN : 979-10-97024-00-0

ISSN : 2417-4211

\section{Référence électronique}

Thomas Mollier, «Ce que les Essais nous apprennent sur les impensés de la philosophie ", Essais [En ligne], Hors-série 3 | 2016, mis en ligne le 26 février 2021, consulté le 18 mars 2021. URL : http:// journals.openedition.org/essais/6819; DOI : https://doi.org/10.4000/essais.6819 


\section{Ce que les Essais nous apprennent sur les impensés de la philosophie}

\section{Thomas Mollier}

Que risque-t-on à lire les Essais en philosophe ? La question réfléchit et circonscrit une expérience inquiète ; celle d'une lecture animée par un doute, travaillée du scrupule de ne pouvoir donner ses titres et dire au nom de quoi, à la fin, les Essais seraient une œuvre philosophique. La réponse qu'elle appelle, ou si l'on veut, l'entreprise qui vise à penser cette expérience, relève par nature du discours méthodologique. Ceci est donc un article de méthode.

L'exposé d'une méthode manque toujours d'à-propos. Qu'il vienne après usage, on le dira abstrait et fastidieux ; mais s'il le devance, on le jugera aride et programmatique, sinon incantatoire ${ }^{1}$. À défaut de remède radical, l'exposition d'une méthode conjure son propre péril lorsqu'elle franchit le point de bascule qui sépare la pensée d'une expérience de l'expérience de cette pensée². L'exposition de la méthode se confond alors avec l'expérience de son sol et de ses contours propres : la méthode se constitue en énonçant ses raisons. En ce sens précis, les pages qui suivent revendiquent un caractère expérimental.

Il y a donc une situation initiale : le regard d'un lecteur philosophe sur le massif textuel que constituent les Essais. Pour que cette situation puisse se déployer en l'expérience d'une méthode philosophique, il est formellement nécessaire que deux conditions soient réunies : on appellera lecteur philosophe celui qui (1) cherche à fonder en raison l'application du prédicat " philosophique » à tout ou partie des Essais et (2) soutient, au moins à titre de principe régulateur pour son interprétation, que le texte présente un sens objectif vers

1 D'importants développements de cet article présentent un caractère indubitablement programmatique. Je prie le lecteur de croire qu'ils s'appuient pourtant sur de minutieuses études, dans les Essais, de " lieux de cohérence " abritant des " micro-réseaux " promoteurs de philosophèmes - voir infra. Ces études locales sont aujourd'hui en cours d'achèvement et de prochaines publications ne manqueront pas de donner substance à ce qui ne peut ici qu'apparaître insuffisamment étayé.

2 Il semble que c'est un point de bascule de ce genre qui sépare, chez Descartes, les Regulae ad directionem ingenii et le Discours de la méthode. 
lequel il est possible de tendre en développant des arguments en faveur de telle lecture plutôt que de telle autre. À ces deux conditions seulement, l'exposition d'une méthode philosophique devient critique de la philosophie : à mesure que le lecteur philosophe fait usage du principe de raison pour établir ce qui est philosophique dans les Essais, se clarifient en effet les fondations mêmes de la philosophie en tant, du moins, qu'elle pose au texte de Montaigne la question de sa philosophicité. Cette démarche critique est celle qui est ici poursuivie.

Cette entreprise n'est pas neutre ; elle suppose formellement un ancrage philosophique dans une stratégie de nature critique. Pour autant, le concept de "philosophie " ici défini ne doit en aucun cas être investi d'une valeur dogmatique ou d'une teneur prescriptive ${ }^{3}$; sa seule vertu dans ces lignes est opératoire en ce sens qu'il a vocation à éclairer à quelles conditions le lecteur des Essais est fondé à parler de "la philosophie de Montaigne ». De ce point de vue le résultat de cette expérience méthodologique intéresse en droit tout lecteur des Essais.

Il s'agit donc ici de mesurer et de penser la résistance des Essais à fournir des raisons suffisantes au lecteur philosophe de leur appliquer, à tout ou partie, le prédicat " philosophique ". La portée de cette expérience de pensée est directement fonction de la rigueur méthodique qui l'anime. L'exigence critique menace en effet toujours de s'essouffler et, partant, de se trahir ; mais, c'est la valeur heuristique d'une telle méthode qu'il est question ici d'éprouver, et, avec elle, l'exceptionnalité éventuelle des Essais. Deux des recueils d'articles les plus significatifs des quinze dernières années invitent à une telle enquête. Dans le mot liminaire du volume XII des Montaigne Studies, " La Philosophie et Montaigne », Philippe Desan justifie le titre retenu en estimant que le livre de Montaigne appelle à soi seul, une reconfiguration du champ philosophique contemporain ${ }^{4}$. Quant à la référence explicitement freudienne du titre choisi par Jean-Yves Pouilloux pour le numéro 41-42 du Bulletin de la Société des Amis de Montaigne 5 , "Montaigne, malaise dans la philosophie ", elle suggère que poser aux Essais la question de leur propre caractère philosophique pourrait ébranler jusqu'aux fondations mêmes de la philosophie qui s'ose à cette question, et dévoiler ce faisant certaines de ses fondations qu'elle n'aperçoit pas ou ne pense pas encore. En mesurant, dans un va-et-

3 Je n'entends ni affirmer ni imposer dans cet article une conception rigide de la philosophie. J'utilise seulement un dispositif argumentatif critique pour tenter de mieux comprendre la spécificité philosophique des Essais et ce qu'ils peuvent donner à penser, du moins à ceux qui pratiquent une philosophie qui cherche à rendre raison d'elle-même lorsqu'elle interprète des textes.

4 Philippe Desan, «La Philosophie et Montaigne », Montaigne Studies, vol. XII, 2000, p. 3-4.

5 Jean-Yves Pouilloux (éd.), "Montaigne, malaise dans la philosophie ", Bulletin de la Société des Amis de Montaigne, $\mathrm{n}^{\circ}$ 41-42, janvier-juin 2006. 
vient critique, ce qui advient au point de rencontre entre le lecteur philosophe et les Essais, on entend donc se donner les moyens de surmonter ce malaise et mettre en lumière ce que la philosophie ne pense jamais assez lorsqu'elle entreprend de ressaisir l'étrange philosophie du livre de Montaigne.

\section{La résistance des Essais comme contrainte pour la lecture philosophique}

En quoi les Essais présentent-ils une résistance pour le lecteur philosophe? En premier lieu et avant tout, le langage des Essais est le langage ordinaire. Cela ne signifie pas que Montaigne n'emploie pas, à l'occasion, des concepts indubitablement inscrits dans l'histoire de la philosophie - témoin, le titre cicéronien du chapitre «De l'utile et de l'honnête » (III, 1). Cela ne signifie pas non plus que Montaigne ne recoure pas, de temps à autre, à la forme syllogistique $^{6}$. Il s'agit simplement de souligner combien les Essais se démarquent des modalités les plus courantes d'auto-constitution des discours philosophiques ${ }^{7}$ en renonçant à fonder les catégories du langage dans lequel ils se déploient. D'un point de vue philosophique, l'entreprise des Essais n'a pas de fondement ; seulement un commencement dont Montaigne fait état au chapitre «De l'oisiveté » (I, 8). La pratique d'enregistrement, de « mise en rolle » (I, 8, $33 \mathrm{~A})^{8}$, définit un régime de production textuelle dont le principe d'organisation semble extérieur à ses effets : ce n'est pas la seule nécessité logique qui conduit Montaigne d'une proposition à une autre. Dit autrement, la philosophie de Montaigne n'est pas systématique. Or, si cette condition non-systématique de la philosophie montanienne suffit à justifier les approches historiques des Essais - qu'elles soient de nature sociologique ou philologique -, le lecteur philosophe, quant à lui, se voit contraint de composer avec un matériau hétérogène aux procédures qu'il pratique. La philosophie ne se reconnaît donc jamais immédiatement dans les Essais.

À contrario, face au langage ordinaire des Essais, il incombe au philosophe d'accomplir un travail de distinction entre le philosophique et le non-philosophique. Le résultat de ce travail, ce qu'on appelle une lecture philosophique des Essais, est une traduction des Essais dans le régime discursif de la philosophie :

6 George Hoffmann, «Fonder une méthode à la Renaissance : Montaigne et ses professeurs de philosophie (I) ", Bulletin de la Société des Amis de Montaigne, juillet-décembre 1990, p. 31-57 ; Id., "Fonder une méthode à la Renaissance (II partie) ", Bulletin de la Société des Amis de Montaigne, juillet-décembre 1991, p. 45-62.

7 Dominique Maingueneau, "L'énonciation philosophique comme institution discursive ", in Frédéric Cossuta (éd.), "L'analyse du discours philosophique ", Langages, $29^{\circ}$ année, n 119 , 1995, p. 40-62.

8 Toutes nos références aux Essais, incorporées au texte, renvoient à l'édition de Pierre Villey et V.-L. Saulnier, Paris, Presses Universitaires de France, 1965. 
traduction censée restituer la philosophie de Montaigne. Cette opération de traduction des Essais se heurte néanmoins très vite à une difficulté de taille, liée précisément à l'emploi par Montaigne du langage ordinaire. Le langage ordinaire est un langage inégalement déterminé. Il n'est pas d'une constante précision, sa référence n'est pas toujours rigoureusement identifiable; certains termes tendent au concept alors que d'autres demeurent imprécis, incertains, vagues. Il n'est pas non plus toujours cohérent : le même terme peut, dans un certain contexte recevoir une détermination sans équivoque et présenter un sens plus flottant dans d'autres. Ainsi les quatre-vingt-quatre occurrences du terme "désir » réparties à travers les Essais ne renvoient-elles pas toujours au concept de "désir " tel qu'il se manifeste, au chapitre "Que nostre desir s'accroît par la malaisance " (II, 15), dans son rapport graduel avec ce qui lui résiste. Considérées à l'échelle de l'œuvre, ces variations nourrissent l'impression de désordre, voire de contradiction qu'éprouve le lecteur des Essais. Cette confusion constitue une entrave majeure pour la détermination des contenus philosophiques à laquelle le lecteur philosophe s'efforce de procéder'.

D'où un changement de focale, dont les lectures philosophiques des Essais sont coutumières, qui consiste à rechercher le projet, le questionnement ou la pratique philosophique unifiante dont l'hétérogénéité radicale des Essais serait le symptôme. Ce changement demande à être questionné dans ses fondements. Les Essais s'offrent-ils aisément à une appréhension par le biais d'une unité théorétique ou pratique de rang supérieur ? Cela ne semble pas être le cas. Il existe, certes, une forme d'unité dans les Essais, que Montaigne ne cesse de revendiquer. Cette unité caractérise, en propre, les Essais de Michel Seigneur de Montaigne, livre "consubstantiel à son autheur " (II, 18, 665 C), dont le cours et le " trein " (III, 2, 806 B) ne font qu'un avec celui du " moy " empirique du Bordelais. Montaigne n'est pas un "faiseur de livres " (I, 20, $90 \mathrm{C}$ ) et son livre n'est pas un artefact, seulement l'exercice d'une écriture témoignant de soi. De cette unité proclamée, le philosophe conclurait volontiers que les fluctuations, les contradictions, les variations conceptuelles desquelles le lecteur des Essais est familier, s'inscrivent dans un projet général de montrer, en acte, la mobilité universelle et l'évanescence fondamentale des choses. Cette conclusion philosophique serait pourtant imprudente, sinon péremptoire.

Imprudente, si elle repose sur l'idée que les Essais pourraient être compris comme l'expression rhétorique d'une philosophie. Certes, l'unité avouée du locuteur des Essais autorise et encourage les lectures rhétoriques de Montaigne. Mais poser une équivalence, à l'échelle de l'œuvre, entre rhétorique et philosophie en acte supposerait de pouvoir dégager une théorie

9 Sur le défi que constitue le " désordre " des Essais pour la lecture, voir Jean-Yves Pouilloux, Lire les « Essais» de Montaigne, Paris, Maspero, 1969, III, p. 43-60. 
proprement montanienne de la communication de la philosophie ${ }^{10}$, à même d'assigner à chaque passage un statut dans une stratégie d'ensemble de nature philosophique.

Or, une telle théorie demande encore à voir le jour. Il lui faudrait rendre raison de l'inefficacité, brocardée par Montaigne dans «De la vanité » (III, 9), de « ces poinctes eslevées de la philosophie sur lesquelles aucun estre humain ne se peut rasseoir » (III, 9, 989 B) et elle devrait surtout justifier l'impuissance, soulignée quelques lignes plus loin dans le même chapitre, du discours philosophique le mieux composé, le plus éloquent, à « chatouiller ou poindre [notre] conscience ». Sans doute pourrait-elle alors invoquer une stratégie du locuteur destinée à mieux transmettre encore sa propre philosophie ; soit, mais elle ne le pourrait qu'en présupposant la dimension philosophique de cette posture, c'est-à-dire en faisant d'emblée des Essais un ouvrage philosophique. Elle présupposerait ce qu'elle prétend chercher : pure pétition de principe. Que la question de la sincérité de Montaigne puisse, en contexte, éclairer la compréhension philosophique de tel ou tel passage, cela ne fait aucun doute ; mais aucune conclusion architectonique sur cette question ne devrait pouvoir servir d'argument philosophique, puisqu'en rigueur, aucun argument issu du texte ne permet de qualifier de philosophique la relation du locuteur à son propre discours.

Plus péremptoire encore serait sans doute l'assignation d'un contenu philosophique aux Essais à partir d'un questionnement ou d'une intention ordonnante qui leur seraient extérieurs. Il convient ici de marquer une distinction. On peut évidemment philosopher à ses aises avec les Essais, et à bon droit. Comme on peut le faire avec L'homme sans qualités de Robert Musil, ou avec La Route des Flandres de Claude Simon, sans qu'importe la singularité de leurs démarches pour leurs éventuels contenus philosophiques. Mais c'est tout différent ${ }^{11}$ que de tenter d'appréhender en philosophe la philosophie de Montaigne à partir du seul texte des Essais et de ses idiosyncrasies. Si philosopher avec les Essais autorise, jusqu'à un certain point, à importer sans vergogne des considérations philosophiques extérieures au texte, appréhender les Essais en philosophe exige, par différence, de ne pas leur imputer a priori la qualité de philosophique. C'est une condition de la démarche critique que le lecteur philosophe prenne en effet le risque de mettre en question la philosophicité du texte de Montaigne.

10 À l'instar des travaux de Laurent Jaffro à propos de Shaftesbury : Éthique de la communication et art d'écrire. Shaftesbury et les Lumières anglaises, Paris, Presses Universitaires de France, 1998.

11 Sur ce point, Philippe Sabot in Philosophie et Littérature. Approches et enjeux d'une question, Paris, Presses Universitaires de France, 2002, distingue judicieusement entre approches " didactique " et " herméneutique " de la littérature par la philosophie où l'objet textuel n'est qu'illustration ou symptôme et approche " productive » où le lecteur tente de rendre compte des effets produits par le mode singulier d'écriture du texte. 
L'approche philosophique des Essais donne donc lieu à une double difficulté de réglage que synthétisent les deux propositions suivantes : (1) la philosophie n'est pas partout dans les Essais et (2) la philosophie n'est pas le Tout des Essais. La philosophie n'est pas partout dans les Essais parce que le principe de consécution des propositions à l'œuvre dans le texte n'est pas fondé en raison et parce que le langage ordinaire dans lequel se présente le texte est inégalement déterminé. La philosophie n’est pas le Tout des Essais parce que l'unité éthique du livre de Michel Seigneur de Montaigne ne peut être dite philosophique qu'au prix d'une pétition de principe, et parce que le lecteur philosophe, sans contredire son propre questionnement, ne saurait conférer a priori, aux textes qu'il interprète, une teneur philosophique. Ces deux propositions constituent un cadre contraignant pour toute lecture philosophique des Essais en quête de la philosophie de Montaigne. Quelles conséquences ce cadre emporte-t-il au point de rencontre entre la philosophie et les Essais?

\section{La question de l'échelle de lecture comme principe cardinal}

Ni partout dans les Essais, ni le Tout des Essais, la philosophie de Montaigne demande donc à être conjointement et indissolublement identifiée et localisée. Le prédicat " philosophique " ne peut que qualifier des espaces textuels finis, doués d'un début et d'une fin, c'est-à-dire quantitativement définis au sein des Essais ${ }^{12}$. "Philosophique " doit ainsi être dit d'un lieu textuel, quantitativement et qualitativement déterminé. L'identification des philosophèmes dans le texte des Essais requiert donc que soit posée la question : "quand y a-t-il des philosophèmes ? ", c'est-à-dire à la fois et indissolublement " où y a-t-il des philosophèmes ? " et " quelle est leur condition ? ". Cette double question, au principe du nouage entre la philosophie comme méthode et les Essais, il convient de l'appeler question de l'échelle de lecture philosophique des Essais. Y répondre passe par le repérage de lieux de cohérence philosophiques : espaces textuels de longueur variable ${ }^{13}$ qui constituent, promeuvent et abritent en des ensembles solidaires les philosophèmes montaniens.

À quel bouleversement la philosophie se trouve-t-elle en proie lorsqu' elle soumet sa lecture des Essais à la question de l'échelle de lecture philosophique ? Elle se voit conduite à engager un travail de délimitation de soi. Autrement dit, elle doit faire place à quelque chose qui lui est extérieur et pourtant pas accessoire.

12 L'importance de la délimitation d'espaces textuels pour ressaisir la pensée de Montaigne a été soulignée, exemple à l'appui, par Olivier Guerrier in "Cadre et figure de pensée ", Bulletin de la Société des Amis de Montaigne, $\mathrm{n}^{\circ}$ 41-42, janvier-juin 2006, p. 131-141.

13 Le chapitre ne saurait tenir lieu d'unité que par provision : voir, notamment, JeanYves Pouilloux, Lire les " Essais" de Montaigne, op. cit., p. 39. 
À cette aune, les Essais apparaissent comme un lieu de crise pour la philosophie. Dans une approche placée sous la question cardinale de l'échelle de lecture philosophique, le prédicat " philosophique " vient en effet qualifier des éléments quand et parce quills sont positionnés d'une certaine manière. Cela signifie que les éléments philosophiques, les philosophèmes, doivent leur qualité de " philosophique " à leur voisinage, à la proximité qu'ils entretiennent avec d'autres éléments textuels, philosophiques et surtout nonphilosophiques. Guidée par la question de l'échelle de lecture, une approche philosophique rigoureuse des Essais semble donc devoir coordonner le philosophique et le non-philosophique pour pouvoir accéder à la philosophie de Montaigne. Le contenu philosophique des Essais requiert aussi pour être saisi la médiation d'une attention extra-philosophique.

Le repérage de la philosophie dans les Essais sollicite donc l'ouverture du regard du lecteur philosophe à des effets attestés dans le texte mais d'ordinaire ignorés. La conversion du regard du philosophe lecteur des Essais s'indique dans la prise en compte de tout ce que l'on appelle généralement les effets littéraires : les effets de style, et avant tout les figures, les effets du discours, notamment l'ironie et les effets sonores, par exemple les paronomases. Les effets littéraires ne sont plus alors ni les atours séduisants d'une pensée qu'il s'agirait de dévoiler, ni le miroir d'une pensée qui présiderait à leur disposition ; ils concourent activement à la construction de philosophèmes qui ne sont tels que dans le texte. L'hétérogénéité radicale des Essais oblige la philosophie à un décentrement et l'interprète à un détour aux termes desquels seulement il doit être possible d'espérer découvrir la philosophie de Montaigne.

\section{Le paradoxe du Tas, paradoxe de la philosophie de Montaigne}

Le décentrement imposé à la philosophie se comprend au fond comme un problème critique adressé à la philosophie quant à ce qu'elle est : l'approche philosophique des Essais peut-elle encore trouver des philosophèmes dans les Essais si les conditions de leur philosophicité sont une conjonction indissoluble entre non-philosophique et philosophique en un lieu déterminé à la fois quantitativement et qualitativement ? Double problème de reconnaissance de soi et d'identité, la question, face aux Essais, s'énonce plus simplement ainsi : la philosophie est-elle encore philosophie quand elle est intrinsèquement tributaire de variations quantitatives? Ou, en termes méthodologiques : une approche philosophique peut-elle reconnaître la philosophie montanienne si celle-ci dépend en substance des effets textuels ? Par les problèmes qu'ils lui posent, les Essais mettent la philosophie au défi : ils font de la philosophie l'objet d'un de ses plus vieux paradoxes, le paradoxe du sorite. Pour l'interprète philosophe, la philosophie de Montaigne se trouve dans la position du tas du sorite, un paradoxe à surmonter. 
Diogène Laërce ${ }^{14}$ attribue ce paradoxe à Euboulide de Milet, philosophe du IV siècle affilié à l'école mégarique. Il s'énonce ainsi : un grain n'est pas un tas; si on ajoute un grain à un non-tas, on ne forme pas un tas ; par conséquent, on peut augmenter autant que l'on veut la quantité de grains sans jamais parvenir à constituer un tas. À l'inverse, un tas auquel on retire un grain reste un tas, si bien qu'un seul grain est encore un tas : on peut diminuer la quantité de grains autant que l'on veut sans jamais parvenir à constituer un non-tas. Apparaît aussi une contradiction qui soulève la question suivante : quand a-t-on devant soi un tas ? c'est-à-dire à la fois et indissolublement pour quelle quantité de grains et dans quel contexte ? La question est très précisément la même que celle qui se pose au lecteur philosophe à propos de la philosophie dans les Essais. Le problème ici en jeu, pour la philosophie de Montaigne comme pour le tas est celui de l'articulation de la quantité et de la qualité, ou, plus exactement, celui du lien et du passage de l'une à l'autre.

Pour mieux fonder l'homologie entre l'énigme du tas et l'énigme de la philosophie dans les Essais, il convient encore de préciser en quoi peut consister la quantité des lieux de cohérence qu'il incombe à la philosophie de rechercher. Cette quantité qui serait l'analogue du nombre de grains ne saurait se comprendre que comme la densité au voisinage d'un terme susceptible de recevoir la qualification de philosophème. Un exemple suffit à le concevoir : au chapitre "Que nostre desir s'accroît par la malaisance " (II, 15), Montaigne évoque bien entendu le désir, mais aussi, tout à côté, un nombre significatif de termes relevant du même espace thématique, la volonté, le goût, l'amour, la volupté et l'appétit. Si chacun de ces termes est bien sémantiquement distinct, ils relèvent tous d'un champ lexical commun et sont semblables sans être identiques. Or, comme Terence Cave l'a justement montré15, l'abondance des mots, la copia du texte de Montaigne est en soi source de réflexivité. Mieux, leur proximité dans le texte dessine un espace densifié, où par le jeu de leurs petites différences, ces termes se déterminent les uns les autres et acquièrent une stabilité et une validité certaines dans le lieu qui les rapproche. Pour un terme considéré, cette densité de voisinage va donc de pair avec un accroissement en détermination, dont la source est de nature quantitative. La question pour le lecteur est alors de savoir indissociablement à partir de quel degré de détermination et dans quel contexte il se trouve en présence d'un philosophème. Exprimé en ces termes, le problème

14 Diogène Laërce, Vies, doctrines et sentences des philosophes illustres, II, 108, où il est question d'Euclide de Mégare. Montaigne avait-il connaissance de ce paradoxe par la lecture des Académiques de Cicéron ? C'est ce qu'estime Luiz Eva, in " Montaigne et les Academica de Cicéron ", Astérion [en ligne], 11|2013.

15 Terence Cave, The Cornucopian Text: Problems of Writing in the French Renaissance, Oxford, Clarendon Press, 1979, p. 271-321. 
de la philosophie des Essais est bien le même que le problème du sorite. Tout comme le sorite interroge une succession de groupes de grains candidats à la qualification de "tas ", le lecteur philosophe des Essais interroge une succession de termes candidats à la qualification de "philosophique ". Dans les deux cas, la question n'a de sens que dans la répétition puisqu'elle appelle une réponse par comparaison renouvelée entre deux quantités candidates; là entre deux nombres de grains dans une expérience dont le protocole réitératif garantit la successivité, ici entre deux densités au voisinage d'un terme dans une lecture dont la nature temporelle garantit la successivité. Le problème du sorite qui cherche à définir le tas, comme celui du lecteur philosophe qui cherche à définir la philosophie de Montaigne, est donc de penser comment une différenciation quantitative peut jamais marquer le franchissement d'une limite qualitative. Questionner la limite entre tas et non-tas revient aussi à questionner la limite entre philosophie et non-philosophie dans les Essais.

\section{Solutions philosophiques au paradoxe de la philosophie dans les Essais}

Dès lors, l'examen des principales solutions données par la philosophie au paradoxe du sorite doit pouvoir rendre raison des différents jugements portés sur la philosophie de Montaigne et révéler, en un éclairage critique, certaines dimensions de la philosophie en tant que telle. Par souci d'économie, il ne sera question ici que des solutions apportées au problème au $\mathrm{XX}^{\mathrm{e}}$ siècle par la tradition analytique, qui s'y est ardemment consacrée ${ }^{16}$. Quatre solutions principales semblent se dégager ${ }^{17}$.

La première, très radicale, celle de Bertrand Russell par exemple ${ }^{18}$, consiste à soutenir que le paradoxe du sorite concerne exclusivement le langage naturel. Ce paradoxe mettrait seulement en lumière le caractère sémantiquement "vague » de celui-ci, et permettrait par contraste de distinguer le langage idéal, qui serait seul l'objet de la philosophie. L'application de cette solution à la question de la philosophie dans les Essais conduirait à dénier au texte de Montaigne toute qualité philosophique. Le regard philosophique sur les Essais ne pourrait alors que conclure à l'absence de philo-

16 L'articulation entre quantité et qualité n'a jamais cessé de faire l'objet d'élaborations philosophiques. A titre d'exemples, pour la tradition continentale, voir Jean Theau, "Le rapport quantité-qualité chez Hegel et Bergson ", Philosophiques, vol. 2, n 1, 1975, p. 3-21 ; ou encore Mathieu Horeau, «Saut qualitatif et rationalité. La position philosophique de Kierkegaard " Archives de Philosophie, n 4, 2013, p. 635-649.

17 Pour un état de l'art sur la question, voir Dominic Hyde, "Sorites Paradox ", in The Stanford Encyclopedia of Philosophy, éd. Edward N. Zalta, http://plato.stanford.edu/archives/ win2014/entries/sorites-paradox/.

18 Bertrand Russel, "Vagueness " in The Australian Journal of Philosophy and Psychology, 1923, p. 84-92. 
sophie montanienne ou, à défaut, à la pratique inlassable par Montaigne d'une antiphilosophie de nature thérapeutique ${ }^{19}$. Ce qu'on apprend ici de la philosophie, c'est qu'elle peut d'elle-même reconnaitre, désigner et qualifier sa propre négation.

La deuxième solution, défendue récemment par Timothy Williamson et Roy Sorensen ${ }^{20}$, fait du paradoxe du sorite le révélateur d'un problème épistémologique : il existerait des prédicats dont on pourrait avec certitude dire qu'ils ont des limites sémantiques sans néanmoins pouvoir précisément les établir. On veut dire quelque chose de précis lorsque l'on parle d'un tas, mais on se trouve pourtant dans l'impossibilité d'en donner les limites. Le " philosophique " dans les Essais serait un prédicat de ce genre. On tendrait alors à faire de la philosophie de Montaigne une philosophie manifeste mais impossible à définir, sans concepts ni thèses, c'est-à-dire sceptique de tendance zététique. Cette solution dévoile la propension de la philosophie à se penser comme un régime discursif d'extension illimitée, un dedans sans dehors, garanti par la dynamique propre de la réflexivité.

La troisième solution, la plus répandue dans la seconde moitié du $\mathrm{XX}^{\mathrm{e}}$ siècle $^{21}$, recourt à des logiques multivalentes, c'est-à-dire à des logiques à plus de deux valeurs de vérité, et notamment à des logiques à nombre infini de valeurs où la vérité se présente par degrés ${ }^{22}$. On dirait ainsi de certains prédicats, le tas de l'argument du sorite ou la philosophie dans les Essais, qu'ils sont le cas, ou non, ou plus ou moins. Pareille solution tendrait à faire de la philosophie de Montaigne un scepticisme probabiliste, producteur, à l'occasion de ce que Frédéric Brahami a appelé des " petits concepts ${ }^{23}$ " et capable, peut-être, avec Jean-Yves Pouilloux d'atteindre à une pleine teneur philosophique dans les énoncés formels séparables des énoncés idéologiques $^{24}$. Avec cette solution, la philosophie atteste de sa capacité à se faire critique d'elle-même, en révisant, si nécessaire, un principe aussi fondamental que sa conception de la vérité.

19 Ian Maclean, Montaigne philosophe, Paris, Presses Universitaires de France, 1996, rappelle (p. 17) que Montaigne n'a été inscrit au programme de l'agrégation de philosophie qu'en 1993. Encore ne s'agissait-il alors que de l'" Apologie de Raimond Sebond ". Les Essais dans leur intégralité firent leur apparition au programme en 2006.

20 Timothy Williamson, Vagueness, Londres, Routledge, 1994 ; et R. Sorensen, Vagueness and Contradiction, New York, Oxford University Press, 2001.

21 Voir, par exemple, Michael Tye, "Sorites Paradoxes and the Semantics of Vagueness ", in Philosophical Perspectives : Logic and Language, éd. James Tomberlin, Atascadero, Ridgeview, 1994, p. 189-206.

22 Nicolas J. J. Smith, Vagueness and Degrees of Truth, Oxford, Oxford University Press, 2008.

23 Frédéric Brahami, Le travail du scepticisme, Montaigne, Bayle, Hume, Paris, Presses Universitaires de France, 2000, p. 236-237.

24 Jean-Yves Pouilloux, Lire les "Essais » de Montaigne, op. cit., p. 38-41. 
Ces trois premières solutions, sur lesquelles repose la grande majorité des lectures philosophiques de Montaigne, confortent la philosophie dans ses propres penchants. La résistance de la philosophie des Essais à l'approche philosophique semble en effet aisément surmontée si la philosophie se fie à sa propre puissance qui consiste (1) en sa prérogative de nomination de sa propre négation, (2) en l'extension infinie de la réflexivité qui la caractérise et (3) en sa capacité critique de révision de ses propres normes. Mais toutes ces solutions ont pour point commun de réintroduire réflexivement, à un rang supérieur, les catégories philosophiques mises à mal par la résistance des Essais au regard philosophique. À telle enseigne, qu'en surmontant le caractère paradoxal de la philosophie de Montaigne, la philosophie redevient le Tout des Essais, non plus comme intention ou comme projet, mais parce qu'elle en devient le sens ultime. C'est donc sans doute là philosopher très profondément avec les Essais, mais peut-être pas encore rendre compte rigoureusement de la philosophie qui y réside.

Une quatrième et dernière solution s'offre encore face à la perplexité. Elle repose sur une explication pragmatiste du paradoxe du sorite, telle que développée par exemple par Hans Kamp ${ }^{25}$. L'incapacité à limiter précisément l'extension des prédicats soritiques vient, dans cette perspective, du fait que la limite n'apparait jamais là où on la cherche. Il faut ici faire une expérience de pensée : imaginons-nous face à la série des tas du sorite alignés et classés par ordre de grandeur. Si je les regarde de gauche à droite, deux tas successifs ne sont jamais séparés que par un grain. Si je les regarde dans le désordre, il y a un " tas » de trois grains et un " tas " de dix mille grains. Si j'avais à comparer le tas de trois grains à celui de dix mille, je dirais naturellement que le premier n'est pas un tas, là où le second l'est. Or, l'argument du sorite repose sur l'itération d'une même comparaison, celle entre un tas et un tas privé d'un grain, dont je ne peux conclure que ce grain en moins affecte sa nature de tas. Si je continue de penser que " tas " a un sens, soutient Hans Kamp, c'est en fait parce que je ne compare qu'en contexte, en réinterprétant constamment l'idée de tas à chaque fois que je glisse d'un couple à un autre. Ce glissement est une action qui se déroule dans le temps. En parcourant la série des tas, je ne parviens jamais à dire quand je ne me trouve plus face à un tas parce que je cherche la limite entre tas et non-tas en un couple où je ne saurais jamais la trouver. Ce qui ne veut pas dire qu'elle n'existe pas, comme en atteste mon sentiment dès que j'abandonne le protocole d'itération du sorite et que je compare un " tas » de trois grains à un " tas » de dix mille, ou bien, comme en me mettant à distance, un couple formé d'un tas de trois grains et d'un autre de trois moins un grains avec un couple formé d'un tas de dix mille grains et d'un tas de dix mille moins un grains.

25 Hans Kamp, "The Paradox of the Heap ", in Aspects of Philosophical Logic, éd. Uwe Mönnich, Dordrecht, Reidel, 1981, p. 225-277. 
Que devient cette solution appliquée à la philosophie dans les Essais? Elle engage d'abord à penser la lecture philosophique, à l'instar du jugement sur le tas, comme un acte herméneutique. Ensuite, elle montre que si on cherche à isoler immédiatement un philosophème en le rapportant à son entourage immédiat et qu'on parcourt ainsi le texte des Essais à la recherche de la philosophie de Montaigne, la quête sera vaine, puisqu'on ne trouvera des termes qu'un peu plus déterminés que ceux qui les entourent : on ne trouve pas immédiatement des thèses ou des concepts dans les Essais. Mais, précisément, le degré de détermination d'un terme dans les Essais ne se comprend qu'en lien avec une densité de voisinage. Il doit donc y avoir dans les Essais une expérience de la limite qui n'est pas la limite des philosophèmes, mais celle des lieux de cohérence où se construisent les philosophèmes grâce à et parmi des notions légèrement moins déterminées. Aussi semble-t-il nécessaire de changer d'échelle : ce sont ces lieux et leur densité qu'il faut comparer les uns aux autres. À condition d'abandonner la quête de discerner à l'échelle des termes la limite entre philosophique et non-philosophique dans les Essais, il doit être possible de faire l'expérience de lieux denses où sont susceptibles d'émerger des philosophèmes. Cette limite n'est pas en soi philosophique. Parce que sa limite est non-philosophique, le lieu de cohérence est l'instrument qui permet de saisir, en philosophe, quelque chose d'extérieur et nécessaire tout à la fois à la philosophie de Montaigne. Espace où s'articulent le philosophique et son dehors, il permet de faire l'expérience de la philosophie dans les Essais sans réduire les Essais à la philosophie, c'est-à-dire, au fond, sans faire violence au texte de Montaigne.

\section{La lecture poïétique des Essais et l'impensé de la philosophie}

Quelle expérience de la philosophie de Montaigne est-il alors possible de faire, ainsi décentré, et que cela révèle-t-il de la philosophie qui la questionne?

Le lecteur philosophe, s'il consent au déplacement qui consiste à porter son regard sur des lieux de cohérence devrait commencer par une lecture semblable à celle que promouvait Jules Brody sous le vocable de "lecture philologique " : lecture qui présume "une parité sémantique ", c'est-à-dire accorde a priori à la totalité des mots le même "potentiel signifiant $t^{26}$ ". Cette lecture offre l'expérience du relief des Essais, de leur non-linéarité, et demeure, par là-même, fidèle à la résistance constatée des Essais à la philosophie. Brody la relate en ces termes : "Le discours des Essais considéré en tant que forme, ferait penser à une surface accidentée, couverte de bosses et de creux, allant par monts et par vaux, laquelle, vue de profil ou en coupe transversale, ressem-

26 Jules Brody, "De l'expérience. Essai de lecture intégrale ", in Lire les Essais de Montaigne, éd. Noël Peacock et James Supple, Paris, H. Champion, 2001, p. 27-38. 
blerait à une courbe sinusoïdale de forme irrégulière, dont les hauts et les bas se propagent et se suivent les uns en fonction des autres ". Pareille expérience invite le lecteur à prêter attention aux processus de constitution des points hauts du texte ${ }^{27}$; à la manière, pour ainsi dire, dont se forme le relief dans le texte des Essais. Ce relief est-il nécessairement philosophique ? Sans doute pas, car il est bien entendu dans les Essais des points hauts pathétiques, des points culminants narratifs et des acmés stylistiques ; mais, là où des termes, des syntagmes ou des propositions se trouvent manifestement plus déterminés par leur position dans un lieu dense, il semble légitime de qualifier ces termes et ces propositions de concepts et de thèses philosophiques.

Or, ce qu'il importe ici de noter, c'est que ce sont les mêmes ressorts qui sont à l'œuvre dans la constitution des points hauts philosophiques et extraphilosophiques. Ces ressorts sont des relations entre des éléments du langage des Essais: des relations sémantiques, les champs lexicaux entre autres, des relations figurales, les relations de contiguïté et de similarité que produisent métonymies et métaphores, et des relations sonores, celles par exemple des assonances et allitérations. Ces relations constituent des lieux denses dans le texte de Montaigne, lieux qui sont autant de micro-réseaux qui soulèvent et résolvent chacun un problème philosophique. Si ces réseaux se chevauchent et font des Essais un tuilage, une "marqueterie mal jointe " plutôt qu'une concaténation, il n'empêche que chacun pris individuellement peut se voir assigner des limites claires en fonction de critères relationnels de nature proprement littéraire. Or, la densité de ces lieux est le seul moyen de donner substance à une philosophie qui n'est ni partout dans les Essais ni le Tout des Essais. Les réseaux des Essais ainsi définis apparaissent alors comme l'objet de la quête du lecteur philosophe des Essais. La philosophie de Montaigne se donne aussi comme une philosophie construite, comme le produit de la dimension relationnelle de la langue, de son travail de liaison, de sa puissance de rapprochement.

La philosophie de Montaigne, parce qu'elle n'est rigoureusement saisissable qu'au prix d'un détour, éclaire ce que la philosophie en tant que telle se refuse à penser : la poïétique dont elle dépend. La philosophie n'est pas un régime de vérité mais un éclat ${ }^{28}$ de vérité dont il faut sans cesse considérer les modalités de production : telle est la leçon d'une approche qui questionne la philosophie des Essais. Le langage de la philosophie ni celui des Essais n'est le simple véhicule matériel d'une pensée qui le devancerait ; il est au contraire le milieu où se développe la pensée. Mieux, il est ce milieu comme texte. Par texte, il faut entendre ici configuration du matériau langagier, dans les deux sens, processuel et résultatif du terme. Dire que la philosophie saillit d'une

27 Je ne partage pas l'interprétation exclusivement stylistique qu'en donne Jules Brody, ibid.

28 J'assume le caractère équivoque du terme, à la fois éclair et fragment. 
configuration revient donc à révéler doublement et indissolublement : (1) que la philosophie est tributaire d'un travail et (2) qu'elle advient en un lieu. Elle ne saurait donc jamais fonder à elle seule la validité universelle de son discours, car celui-ci requiert un travail localisé. Ce travail localisé, c'est celui de l'écriture, au sens matériel et spatial du terme. C'est lui dont témoigne sans égale la dynamique de composition des Essais par ajouts, retouches, allongeails ${ }^{29}$; lui encore, dont attestent les marges de l'Exemplaire de Bordeaux et leurs annotations manuscrites. Travail de rapprochement de mots, de phrases, au gré des circonstances, selon " l'occasion, la compagnie, le branle mesme de la voix » (I, $10,40 \mathrm{~B}$ ). Travail sans fondement, mais lucide et non sans résultat, puisque, rapprochés sur la page, les mots se travaillent les uns les autres, se polissent les uns les autres au point de faire saillir, en quelque endroit, un concept, une thèse, éclatants, car fortement déterminés. Si la validité des philosophèmes dans les Essais est seulement locale, leur succès tient à leur universelle disponibilité pour penser et résoudre des problèmes philosophiques. Leur vérité est leur puissance de rayonnement.

\section{La philosophie de Montaigne : un byproduct}

Les Essais donnent à voir une philosophie qui advient dans le travail localisé du langage lorsque, dans l'acte d'écriture, il prend figure sur la page. C'est le travail à l'œuvre dans la configuration qui permet aux mots de Montaigne d'acquérir la qualité de " philosophique ». La philosophie y apparaît donc comme un produit, que seule une investigation attachée à la dimension poïétique des Essais est à même de déceler. Le recours même à la notion de poïse pour qualifier la production philosophique des Essais est néanmoins problématique car il pourrait laisser accroire que la philosophie de Montaigne serait le fruit d'une pure technique, comme le soulier pour le chausseur ou le fer à cheval pour le forgeron. Le poïéticien de la philosophie ne devrait-il pas en effet reconnaître une visée, une intention philosophique aux Essais ? Dans l'affirmative, l'expérience critique ici conduite toucherait là à son terme et il faudrait avouer que le lecteur philosophe des Essais ne peut jamais faire l'expérience de quelque chose d'à la fois rigoureusement extérieur et fondamentalement déterminant pour la philosophie.

Cette conclusion hautement fâcheuse est pourtant largement évitable : c'est Montaigne même qui nous suggère la solution, et plus précisément sa conception de l'action, cette " action sur fond d'indifférence " analysée très justement par Bernard Sève ${ }^{30}$. Montaigne considère l'action comme un

29 Voir André Tournon, Montaigne. La glose et l'essai, Lyon, Presses Universitaires de Lyon, 1983.

30 Bernard Sève, Montaigne. Des règles pour l'esprit, Paris, Presses Universitaires de France, 2007, chap. XIII, p. 333-349. 
rôle (III, 10) et l'écriture des Essais n'y déroge pas. Il s'y prête comme il se prête aux charges politiques, sans s'y donner. La distance intérieure, le jeu en soi qu'il préserve, sa "nonchalance ", sa "franchise ", c'est un espace de liberté qui préserve l'action « de tout épaississement idéologique ou passionnel $^{31}$ ». Cette indifférence est même plus profondément la garante de la réussite et de l'efficace de l'action, de sa " grâce » et de son " honneur » (III, $8,967)$. Montaigne est donc bien loin d'être un faiseur de philosophie et l'acte d'écriture des Essais ne se fait pas lui-même sans ce fond d'indifférence qui évite que le livre s'épaississe ou se fige. N'y a-t-il rien comme un produit dans les Essais? Si, mais un produit paradoxal, un produit que l'on qualifierait volontiers, à la manière de Jon Elster de "byproduct", d' " effet essentiellement secondaire ${ }^{32}$. La philosophie n'est pas visée comme un but dans les Essais ; elle est produite, c'est tout. Montaigne ne colle pas à sa propre action, pas même à sa propre action d'écriture; la philosophie de Montaigne ne se situe pas non plus dans l'immanence de l'acte d'écriture, elle est plutôt le produit éclatant et paradoxalement non visé de sa liberté maintenue.

"De quel regiment estoit ma vie, je ne l'ai appris qu'apres qu'elle est exploitée et employée. Nouvelle figure : un philosophe imprémédité et fortuite!» (II, 12, 546 C) Cette formule bien connue qui vient conclure un ajout en marge de l'Exemplaire de Bordeaux, apparaît maintenant sous un jour nouveau. Parce qu'effet essentiellement secondaire, la philosophie pratique ne se reconnaît qu'après coup, couronnement et «splendeur » d'une action libre, effet réel et pratique de la liberté et franchise de l'agent. Et de même dans le texte : parce qu'effet essentiellement secondaire, la philosophie théorique ne se reconnaît qu'après coup, effet textuel et poïétique de la liberté de l'écriture. Ni désinvolture ici, ni passive complaisance dans cette "nouvelle figure "; une chose seulement, en guise de leçon critique de Montaigne à la philosophie : la philosophie n'est vraie que lorsqu'elle est seconde.

\section{Thomas Mollier}

Université de Lille

UMR 8163 - Savoirs, Textes, Langage

31 Ibid., p. 348.

32 Jon Elster, Le laboureur et ses enfants : deux essais sur les limites de la rationalité, Paris, Éditions de Minuit, 1986, dont la première partie, sous-titrée « essai sur les effets essentiellement secondaires » est la traduction du chapitre 2 de Sour Grapes : Studies in the Subversion of Rationality, Cambridge, Cambridge University Press, 1983, intitulé « States that are essentially by-products ", p. $43-108$. 


\title{
Résumé
}

A quelles conditions est-il possible de parler légitimement de la philosophie de Montaigne? A la faveur d'une démarche critique, l'article fait surgir comme problème cardinal la délimitation de la philosophie dans les Essais. Le paradoxe du sorite sert de paradigme pour penser les solutions disponibles en philosophie et dévoile le travail localisé de la configuration textuelle comme impensé majeur d'un lecteur philosophe désireux de rendre raison de la philosophie de Montaigne, qui apparait in fine comme un by-product.

\section{Mots-clés}

Philosophie, échelle de lecture, délimitation, lieux de cohérence, configuration, byproduct.

\begin{abstract}
What are the conditions necessary to credit Montaigne with a philosophy of his own? This article singles out discipline demarcations as the crucial issue in defining philosophy in the Essais. Examination of the various responses to the Sorites paradox, a formal equivalent to the above-mentioned problem, reveals the localized interplay between textual elements as a determining, yet unwitting and unconsidered factor in Montaigne's philosophy. Philosophy in the Essais shall therefore be considered a by-product.
\end{abstract}

\section{Keywords}

Philosophy, Montaigne, demarcation, cohesive spaces, configuration, by-product. 